\title{
Authorized Vehicle Recognition System
}

\author{
KedarR $^{\mathrm{a}, 1}$, KavirajA ${ }^{\mathrm{b}}$, Manish R $^{\mathrm{b}}$, NiteeshB ${ }^{\mathrm{b}}$, SuthirS ${ }^{\mathrm{c}}$ \\ ${ }^{a, b}$ UG Scholar, Dept of CSE, Panimalar Engineering College, Chennai \\ ${ }^{c}$ Professor, Dept of CSE, Panimalar Engineering College, Chennai
}

\begin{abstract}
The technology is growing and increasing in our day to day life to satisfy the needs of human beings. The system we are going to propose makes the human job easier. Here the YOLO algorithm which is a deep learning object detection architecture is used to detect the number plate of the vehicle. After detecting the number plate it converts the vehicle number to a text format. Then it checks it with the database to see if the vehicle is authorized to enter into the premise or not. This system can be implemented in highly restrained areas such as military areas, government organizations, Parliament, etc. This proposed system has around six stages: Capture Image, Search for black pixels, Image filtering, Plate region extraction, character extraction, OCR for character recognition. The alphanumeric characters are identified using the OCR algorithm. It is then used to compare the obtained result from the YOLO algorithm with the database and then check if the vehicle is allowed to enter the premise or not. This proposed system is simulated and implemented using Python, and it was also tested on real-time images for performance purposes.
\end{abstract}

Keywords. License Plate Detection, Optical Character Recognition, YOLO.

\section{Introduction}

Vehicle Number plate identification is an image processing approach that is used to detect the vehicles using the number plates. This technique is used mainly in security and traffic installations. This technique will help officials to provide security in authorized areas like the military. This application can also be used for monitoring vehicle inroads for speed monitoring, management of parking and etc. This YOLO algorithm can be used in real-time for recognizing the number of plates under normal conditions. The YOLO algorithm works in six stages capturing an image search for black pixels, image filtering, extraction of data, character recognition and verification with the database.This system is inexpensive compared to other existing systems. Besides the robustness, the earlier methods use either edge detection which is computationally expensive or by artificial neural networks which require training a huge amount of data.

\section{Related work}

The usual number plate recognition system such as the Conventional method of identifying a license plate has three phases Localization of license plates, division of characters and Identification of characters. The first phase of the localization of thevehicle number plate is a part of the vehicle detection technique which involves the

\footnotetext{
${ }^{1}$ Kedar.R, Department of CSE ,Panimalar Engineering College, Chennai: 
localization of the vehicle, feature extraction, andvehicle image classification into three different phases. Because pictures might have different pixels and sizes, the traditional way of detecting the vehicle such as the Deformable Parts Model (DPM)[1] which uses a sliding window mechanism of different sizes to getthe candidate localizations from the picture. The candidate localizations are extracted from the object features with the help of the feature extraction algorithm (SIFT) [2] and then it isreported to the SVM [3] for the identification process. To overcome the unwantedcomputation produced by the sized sliding windows the regional convolutional neural network(CNN) first identifies about 2000 to 3000 regional proposals then adopting the $\mathrm{CNN}$ model to get the features from the regional proposals and it finally finishes the categorization by using the SVM. The regional CNN [4] is experiencing two major disadvantages. The first disadvantage is that it requires each and every area to pass through $\mathrm{CNN}$ which also results in a very hugenumber of iterative computational power for each picture. The another major drawback is that it should learn from all the acquired results of the models one by one or separately. This makes the RCNN very hard to train the models to classify the image.

\section{Proposed Method and Result}

The proposed algorithm is broadly divided into six parts:

i. Capture Image

ii. Identify the black pixels

iii. Image filtering

iv. Plate region extraction

v. Character extraction

vi. Optical character Recognition

\section{License Plate Recognition and Authorization}

\subsection{Identification Of Vehicle}

To detect the vehicles traveling on the road from cameras, we suggest that first, our model detects whether it is a vehicle and only after detecting that it is a vehicle it will scan for the license plate of the vehicles. This approach will avoid traffic signs or ads being misidentified as the vehicle's number plate. The existing object identification methods such as the fast RCNN and regional $\mathrm{CNN}$ are using the selective search or sliding window to find the probable targets and then it makes use of the CNN or various other approaches to detect if the approaching object is a vehicle or not. Due to the complexity of the pictures and the size of the object on the road making use of the sliding window may consume more time and can also produce wrong data or information. So we propose the YOLO algorithm to identify or detect the vehicles. The YOLOv2 [8] has 5 max-pooling layers and 19 convolutional layers. It first extracts all the details from the image and then it reduces the dimensions of the picture and it starts to compress the entire image into the max-pooling layers and the convolutional layers. By comparing both the faster regional CNN and Single SMB Detector the YOLOversion2 is more time saving and efficient in real-time scenarios. It also has higher processing speeds. The vehicles before detection are showed in Figure 1. 


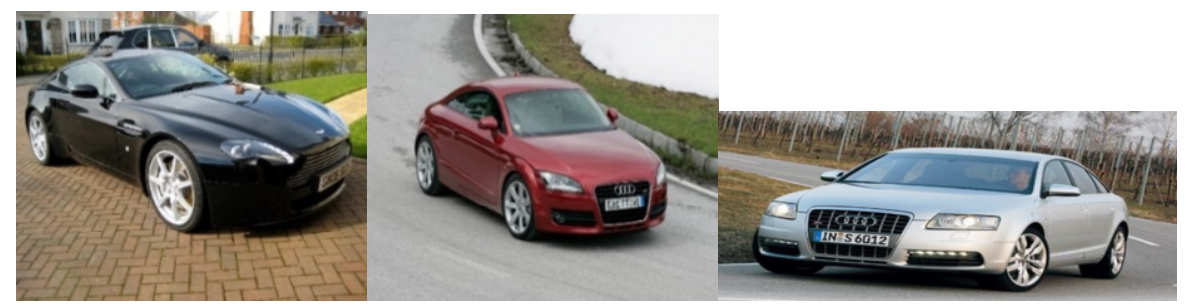

Figure 1. Vehicles before detection

\subsection{Vehicle Number Plate Detection}

This uses the Localization technique to process the vehicle number plate from the given image or live video. The main purpose of this phase is to locate the number plate region of the target from the picture. The quality of every image is mandatory as it increases the accuracy of the character recognition[9]. The number plates are usually of different colors and types such as (Black and Yellow or Black and White). The alphabets and the numbers area placed within the row which changes the horizontal intensity. This provides the changes between the horizontal intensity because the characters present in the rows are expected to produce sharp variations. The purpose that it should produce the sharp variations is that it should differ from the letters and the background. Now the binary image is passed through this technique. After this, the results are fed to the edge detector algorithm to detect the edges of the plate and the characters for the perfection of results. By locating the edges of the picture which reduces the amount of detailing in the image and it helps to filter out the unnecessary information while maintaining the structural properties of the image. The Vehicle and the detection of number plates are showed in Figure 2.

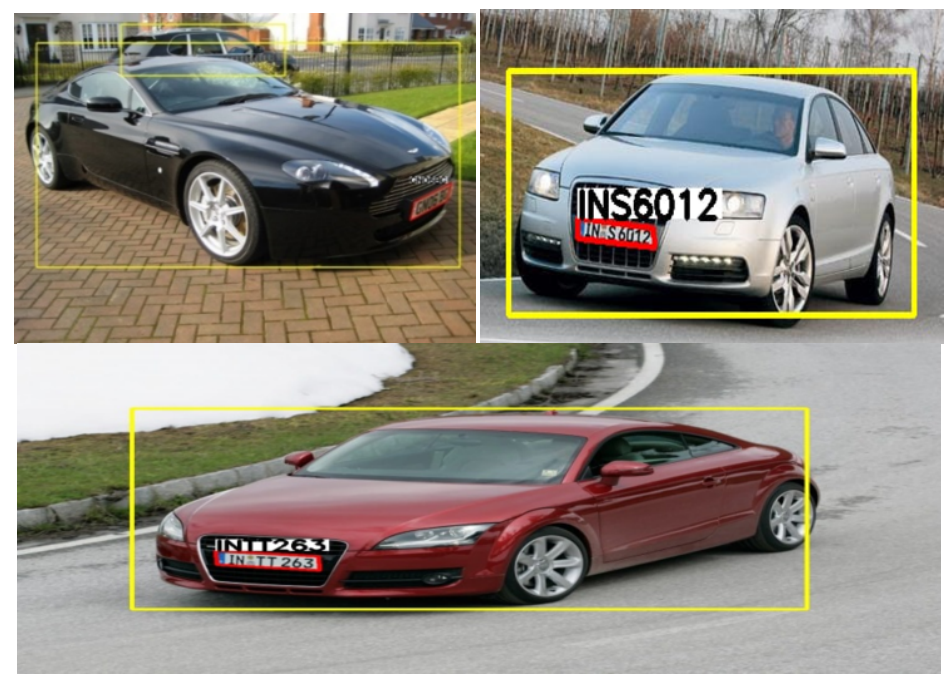

Figure 2. Vehicle and Number Plate Detection 


\subsection{Segmentation of Characters}

After acquiring the images of the number plates of the vehicle, we have to remove the unnecessary area outside the alphabets. The process of segmentation of characters has various steps. At first, the captured picture of the vehicle license plate is converted into a grayscale image and then it is transferred as a binary image to eliminate the distortions and noise in the picture. The horizontal and vertical smearing is used to detect the area of the characters. The next process is to remove the white spaces from the number plate. The horizontal projection is used to locate the spaces and segments of the characters. The adaptive thresholding is used to reinforce the plate before the segmentation process. At last, the vertical projection is made to segregate the characters individually.

\subsection{Optical Character Recognition}

In this stage, characters are recognized accordingly using the Optical Character Recognition (OCR). The Optical Character Recognition uses the template matching to recognize the character present in it. Comparing the templates means that the captured photograph and the template are seen simultaneously and if both the pattern matches it returns the character or else it will iteratively search for the exact similar pattern of the captured image. This method makes use of the database to store all the characters and templates. For all the characters and alphanumeric there is a template that will be stored in the database. The current detail of the character is set to a high contrast and it is enabled with every single format to identify the exact match or the closest information of the character representation. The coordination technique for the layout depends on the target character type, with the help of the standard character format. There are eight objectives for the character coordination. The layout coordination for the acknowledgment of character is done on an Indian Number plate which is a static picture.

\subsection{Number Plate Authorization}

Finally, after all the above processes are made and the characters are recognized using the Optical Character Recognition it is stored in a temporary variable. After recognizing the characters it accesses the database to check whether the vehicle is authorized to enter the premise or not. A database consists of all the required information such as if it is authorized to enter the premise or not. The certain application in which our proposed system is applicable is that in places such as government organizations, parliament, Courts, Toll Booths, Military areas, etc. In certain places like this only a few authorized vehicles are allowed to enter. With the help of our proposed system, we can reduce the manpower and the time efficiency can be increased. This also ensures safety to the premise also. When a vehicle approaches our proposed system will first detect if it a vehicle or not. After that, it will scan for the number plate of the respective vehicle. Then the segmenting of the alphanumericis done and the characters are recognized from it. After the recognition process, the alphanumerical values of the number plate are assigned to a temporary variable and it is cross-checked with the database for the authorization purpose. The authorization using the database is showed in Figure 3. 


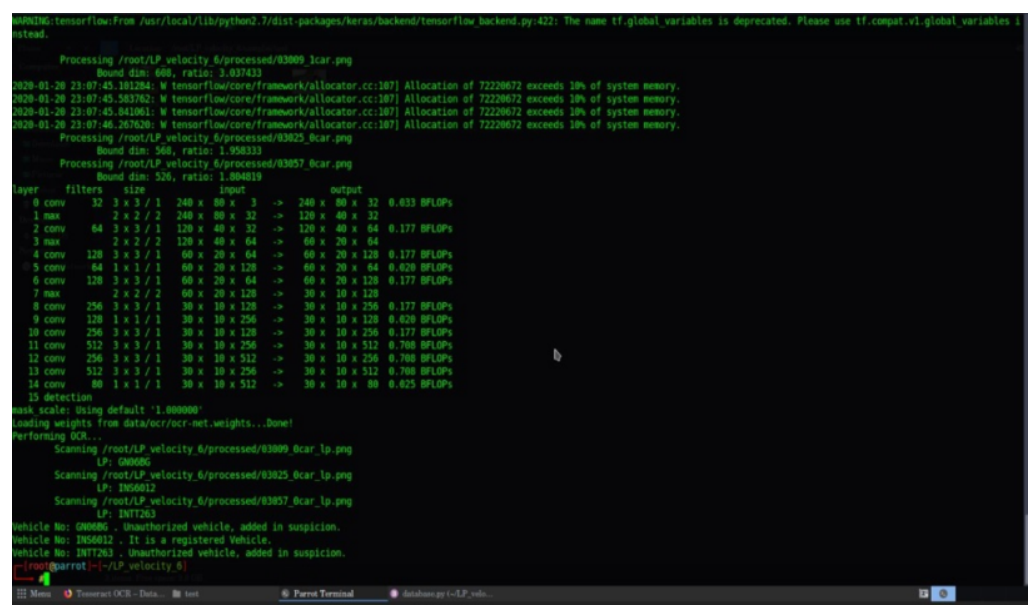

Figure 3. Vehicle Authorization

\section{Conclusions and Further Work}

In this system, the vehicle plates are identified automatically for verification. This system uses a progression of various image processing standards for verifying every vehicle with the database stored in the PC. This system is implemented using python and performance is tested in real-time. The results show that the system detects the number of plates under normal conditions and can be implemented at the entrance of highly restricted areas. The further work in the future would be as a module of face recognition for the additional security for entering into the government or military secured areas. This is a robust real-time scenario system that can be developed and implemented at a low cost.

\section{References}

[1] Girshick.R, Iandola.F, Darrell.T, Malik.J, Deformable Part Models are Convolutional Neural Networks.arXiv preprint arXiv:1409.5403, 2014. In CVPR, 2015.

[2] Lowe.D, "Distinctive image features from scale-invariant keypoints" IJCV, 60 (2), pp. 91-110, 2004.

[3] Dalal.N, Triggs.B .Histograms of Oriented Gradients for Human Detection. Proc. IEEE Conf. Computer Vision and Pattern.

[4] Girshick.R, Donahue.J, Darrell.T, and Malik.J,.Rich feature hierarchies for accurate object detection and semantic segmentation.in Proc. of the 2014 IEEE Conference on Computer Vision and Pattern Recognition (CVPR'14), pp.580-587, 2014.

[5] Girshick.R, Fast R-CNN, in ICCV, 2015.

[6] Rose.K,Title of paper with only first word capitalized.in press. S. Ren, K. He, Girshick.R, Sun.J, Faster R-CNN: Towards Real-Time Object Detection with Region Proposal Networks, in NIPS, 2015.

[7] Redmon.J, Divvala.S, Girshick.R, and Farhadi.A. You only look once: Unified, real-time object detection. arXiv preprint arXiv:1506.02640, 2015.

[8] Redmon.J and Farhadi.A. Yolo9000: Better, faster, stronger. arXiv preprint arXiv:1612.08242,2016.

[9] S.Nishanthini, M.Abinaya, S.Malathi, Smart Video Surveillance System and Alert with Image Capturing using Android Smart Phones, IEEE International Conference on Circuit, Power and Computing Technologies(ICCPCT),2014, p.1714-1722. 